\title{
Recent Development of the Atomic Line List
}

\author{
Peter A. M. van Hoof \\ Royal Observatory of Belgium, Ringlaan 3, B-1180 Brussels, Belgium; p.vanhoof@oma.be
}

Received: 7 May 2018; Accepted: 2 June 2018; Published: 8 June 2018

\begin{abstract}
The Atomic Line List is an online database of wavelengths and transition probabilities of atomic lines. It is primarily set up as a tool to help identify unknown spectral features. This paper briefly describes the web interface, how the line list is constructed, and what development is currently being undertaken for the next release.
\end{abstract}

Keywords: atomic data; spectroscopy; chemical abundances; oscillator strengths; atomic and molecular databases

\section{Introduction}

The Atomic Line List is an online database of wavelengths and transition probabilities of atomic lines. It is primarily set up as a tool to help identify unknown spectral features. It was created in January 1996 as part of the preparations for the ISO mission. The SWS wavelength calibration team was hampered by a severe lack of line lists in the infrared. Therefore, a new line list was created from first principles by using a collection of known energy levels in all ionization stages of all elements up to zinc. A set of allowed and forbidden transitions between these levels was then created using the appropriate selection rules. This allowed a mostly complete line list to be created. The list was initially restricted to the ISO wavelength range, but was quickly extended to a much wider range with no lower limit in wavelength and an upper limit of $1000 \mu \mathrm{m}$. Subsequent extensions were aimed at adding more elements and new transition probability data sets. The latest development version (2.05b21) contains approximately 1.72 million allowed, intercombination and forbidden atomic transitions of elements up to krypton. There are currently no plans to include molecular transitions.

\section{The Web Interface}

The Atomic Line List is available online at http://www.pa.uky.edu/ peter/atomic. Note, however, that this version has not been maintained for many years as all effort now goes into creating the next release. On irregular intervals, beta versions of the upcoming release are being created, which are available at http:/ / www.pa.uky.edu/ p peter/newpage. At the time of writing, this is viewed as the preferred version. The web interface is set up to help the user identify unknown lines in observed spectra. This comprises absorption as well as emission-line spectra of any type of astronomical object and even laboratory spectra. Examples would be stellar atmosphere spectra, nebular spectra of H II regions or planetary nebulae, active galactic nuclei, etc. The selection tool consists of a number of options, each of which has a default value, which is as inclusive as possible. This means that, if none of the options are changed, the complete data set is returned, truncated to 500 lines. The user can then impose restrictions to narrow down the search, e.g. one or more wavelength ranges, a range of elements and/or ions, a minimum abundance, a range of energies of the lower and/or upper level, a minimum line strength, etc. The minimum abundance entered by the user is compared to the standard solar abundances [1] with an optional correction applied for depletion into grains $[2,3]$. The latter would be appropriate for gas in the interstellar medium. The element abundance is used as a simple proxy for the line strength. Note, however, that other factors play an 
important role too, such as the ionization balance, the level populations in the ion, and the transition probability of each line.

The output can also be customized. This allows certain fields to be included or excluded as well as to choose in what way the atomic data should be presented. It is possible to obtain the output in HTML, plain ASCII, or as a $\mathrm{AT}_{\mathrm{E}} \mathrm{X}$ table for inclusion in a paper. The units for the line wavelengths and the level energies can be chosen. The default behavior is to give all wavelengths in vacuum (unlike the NIST Atomic Spectra Database [4]), with air wavelengths as an option. The reason for this choice is that using air wavelengths would impose a default restriction as the wavelengths of the lines are derived from the difference of the level energies (see Section 4) and the refractive index formula by Peck and Reeder [5] used for the conversion to air wavelengths cannot be used below $185 \mathrm{~nm}$. When air wavelengths are selected, the result of the search will be restricted to wavelengths $\geq 200 \mathrm{~nm}$.

\section{A Sample Query}

Table 1 shows the result of a simple query listing all the O II lines in the region between 210 and $211 \mathrm{~nm}$ (in air). By clicking on a term field in the browser, a listing of the complete multiplet that line belongs to can be obtained (in the same format as the original output, see Table 2). Note that the multiplet search has no wavelength restriction, regardless of what was stated in the original query.

Table 1. Sample output from a simple query. The first column gives the laboratory wavelength in air (in $\mathrm{nm}$ ), the second column the species, the third column the transition type, the fourth column the term of the lower and upper level, the fifth column the total angular momentum of the lower and upper level, the sixth column the transition probability (in $\mathrm{s}^{-1}$ ), the seventh column the transition probability literature source, the eighth column the energy of the lower and upper level (in $\mathrm{cm}^{-1}$ ), and the ninth column the literature source for the level energies. Entries $x . x x+y$ stand for $x . x x \times 10^{y}$.

\begin{tabular}{|c|c|c|c|c|c|c|c|c|}
\hline LAB WAVL AIR (nm) & SPC & TT & TERM & $\mathrm{J}_{\mathrm{i}}-\mathrm{J}_{\mathrm{k}}$ & $A_{k i}\left(s^{-1}\right)$ & TPF & LEVEL ENERGY $\left(\mathrm{cm}^{-1}\right)$ & REF \\
\hline 210.00707 & O II & E1 & $4 \mathrm{D}^{\mathrm{o}}-4 \mathrm{~F}$ & $5 / 2-7 / 2$ & $1.40+6$ & 3 & $206,877.86-254,480.20$ & ASD \\
\hline 210.00796 & O II & E1 & $4 \mathrm{D}^{\mathrm{o}}-4 \mathrm{~F}$ & $3 / 2-5 / 2$ & $1.22+6$ & 3 & $206,786.29-254,388.42$ & ASD \\
\hline 210.07247 & $\mathrm{O}$ II & E1 & $4 \mathrm{D}^{\mathrm{o}}-4 \mathrm{~F}$ & $7 / 2-9 / 2$ & $1.63+6$ & 3 & $207,002.48-254,590.00$ & ASD \\
\hline 210.12625 & O II & E1 & $2 D^{\circ}-2 D$ & $5 / 2-3 / 2$ & $7.34+5$ & 3 & $211,712.73-259,288.07$ & ASD \\
\hline 210.12829 & O II & E1 & $2 D^{\circ}-2 D$ & $5 / 2-5 / 2$ & $6.85+6$ & 3 & $211,712.73-259,287.61$ & ASD \\
\hline 210.23238 & O II & E1 & $4 \mathrm{D}^{\mathrm{o}}-4 \mathrm{~F}$ & $3 / 2-3 / 2$ & $4.56+5$ & 3 & $206,786.29-254,337.61$ & ASD \\
\hline 210.41281 & O II & E1 & $4 \mathrm{D}^{\mathrm{o}}-4 \mathrm{~F}$ & $5 / 2-5 / 2$ & $3.96+5$ & 3 & $206,877.86-254,388.42$ & ASD \\
\hline 210.43594 & $\mathrm{O} \mathrm{II}]$ & E1 & $4 \mathrm{P}^{\mathrm{o}}-2 \mathrm{D}$ & $3 / 2-5 / 2$ & & & $208,392.26-255,897.59$ & ASD \\
\hline 210.47372 & $\mathrm{O} \mathrm{II]}$ & E1 & $4 \mathrm{P}^{\mathrm{O}}-2 \mathrm{D}$ & $1 / 2-3 / 2$ & & & $208,346.10-255,842.91$ & ASD \\
\hline 210.55835 & O II & E1 & $4 \mathrm{D}^{\mathrm{o}}-4 \mathrm{~F}$ & $7 / 2-7 / 2$ & $2.32+5$ & 3 & $207,002.48-254,480.20$ & ASD \\
\hline 210.63810 & O II & E1 & $4 \mathrm{D}^{\mathrm{o}}-4 \mathrm{~F}$ & $5 / 2-3 / 2$ & $3.24+4$ & 3 & $206,877.86-254,337.61$ & ASD \\
\hline 210.67847 & O II] & E1 & $4 \mathrm{P}^{\mathrm{O}}-2 \mathrm{D}$ & $3 / 2-3 / 2$ & & & $208,392.26-255,842.91$ & ASD \\
\hline 210.84407 & O II] & E1 & $4 \mathrm{P}^{\mathrm{O}}-2 \mathrm{D}$ & $5 / 2-5 / 2$ & & & $208,484.20-255,897.59$ & ASD \\
\hline 210.96622 & O II & E1 & $4 \mathrm{D}^{\mathrm{o}}-4 \mathrm{~F}$ & $7 / 2-5 / 2$ & $1.54+4$ & 3 & $207,002.48-254,388.42$ & ASD \\
\hline
\end{tabular}

In this particular example, the energy levels came from Martin et al. [6], while the transition probabilities came from the Opacity Project [7]. Many other sources are also present in the line list that are not listed here. 
Table 2. Sample output from a multiplet query obtained by clicking on the first term field shown in Table 1 . The columns and entries have the same meaning as in Table 1.

\begin{tabular}{ccccccccc}
\hline LAB WAVL AIR $(\mathbf{n m})$ & SPC & TT & TERM & $\mathbf{J}_{\mathbf{i}}-\mathbf{J}_{\mathbf{k}}$ & $\mathbf{A}_{\mathbf{k i}} \mathbf{s}^{-\mathbf{1}} \mathbf{)}$ & TPF & LEVEL ENERGY $_{\left(\mathbf{c m}^{-\mathbf{1}}\right)}$ & REF \\
\hline 209.98716 & O II & E1 & $4 \mathrm{D}^{\circ}-4 \mathrm{~F}$ & $1 / 2-3 / 2$ & $1.14+6$ & 3 & $206,730.76-254,337.61$ & ASD \\
210.00707 & O II & E1 & $4 \mathrm{D}^{\circ}-4 \mathrm{~F}$ & $5 / 2-7 / 2$ & $1.40+6$ & 3 & $206,877.86-254,480.20$ & ASD \\
210.00796 & O II & E1 & $4 \mathrm{D}^{\circ}-4 \mathrm{~F}$ & $3 / 2-5 / 2$ & $1.22+6$ & 3 & $206,786.29-254,388.42$ & ASD \\
210.07247 & O II & E1 & $4 \mathrm{D}^{\circ}-4 \mathrm{~F}$ & $7 / 2-9 / 2$ & $1.63+6$ & 3 & $207,002.48-254,590.00$ & ASD \\
210.23238 & O II & E1 & $4 \mathrm{D}^{\circ}-4 \mathrm{~F}$ & $3 / 2-3 / 2$ & $4.56+5$ & 3 & $206,786.29-254,337.61$ & ASD \\
210.41281 & O II & E1 & $4 \mathrm{D}^{\circ}-4 \mathrm{~F}$ & $5 / 2-5 / 2$ & $3.96+5$ & 3 & $206,877.86-254,388.42$ & ASD \\
210.55835 & O II & E1 & $4 \mathrm{D}^{\circ}-4 \mathrm{~F}$ & $7 / 2-7 / 2$ & $2.32+5$ & 3 & $207,002.48-254,480.20$ & ASD \\
210.63810 & O II & E1 & $4 \mathrm{D}^{\circ}-4 \mathrm{~F}$ & $5 / 2-3 / 2$ & $3.24+4$ & 3 & $206,877.86-254,337.61$ & ASD \\
210.96622 & O II & E1 & $4 \mathrm{D}^{\circ}-4 \mathrm{~F}$ & $7 / 2-5 / 2$ & $1.54+4$ & 3 & $207,002.48-254,388.42$ & ASD \\
\hline
\end{tabular}

\section{Construction of the Line List}

The emphasis of the atomic line list is to aim for complete wavelength coverage. This is the main advantage over the NIST ASD data set. The coverage is achieved by starting with a set of observed energy levels. These are read by a computer program which then applies a set of selection rules to derive a set of transitions between these levels. This avoids any wavelength bias, but has the disadvantage that the quantum numbers of the levels need to be fully known to apply the selection rules (around 2.5\% of all observed levels only have a partial set of known quantum numbers or are rejected because they contain typos). The wavelengths of the lines are derived from the difference of the level energies. These are known as Ritz wavelengths and they can be either more or less accurate than observed wavelengths (depending on how many lines connect to lower and upper level). Especially for forbidden lines the accuracy of Ritz wavelengths can be less than observed wavelengths due to the fact that the splitting of the levels cannot be measured directly in laboratory experiments. Thus far, only a very limited effort has been undertaken to include observed wavelengths.

Once the line list has been constructed, it is combined with theoretical transition probability calculations from various sources. To do this, each data source gets assigned a priority based on the perceived quality of the work and the datum from the highest priority source is used. Data from different sources are not averaged. If any of these calculations contain transitions that are not present in the line list constructed thus far, they are automatically added.

This process is very fast, allowing each release to be constructed directly from the original data. This makes the setup very flexible and allows errors to be fixed easily or new features to be added when the need arises.

\section{Current Development}

The original version of the line list was constructed using a Fortran program. This program was constructed based on the assumption that the line list was limited to elements up to zinc and the code was also poorly designed in other ways. This severely hampered further development. Hence, a new program was written from scratch in $\mathrm{C}++$ that is much more flexible. This program is now in the final stages of testing.

With the new program, it is possible to extend the list to heavier elements. The first release will go up to krypton, and later also fifth and sixth row elements will be added. Besides this development, the effort to update the energy level data will continue. In addition, the set of transition probabilities is being significantly extended. Data from large compilations such as the MCHF/MCDHF database by C. Froese-Fischer et al. and the semi-empirical $\log (\mathrm{gf})$ data from R.L. Kurucz are being included. However, data from other calculations for individual ions will also be included. Although this is not the highest priority, the addition of experimental data (especially wavelengths) is foreseen for the future. Eventually, this should make the list also useful for other purposes, e.g. as a data set for radiative transfer calculations. 
The author welcomes submission of data sets with new level energy or transition probability data. These can be sent to the email address listed above.

Funding: This research received no external funding.

Acknowledgments: The author thanks the University of Kentucky for hosting the web site.

Conflicts of Interest: The author declares no conflict of interest.

\section{References}

1. Asplund, M.; Grevesse, N.; Sauval, A.J. The Solar Chemical Composition. In Cosmic Abundances as Records of Stellar Evolution and Nucleosynthesis; Barnes, T.G., III, Bash, F.N., Eds.; Astronomical Society of the Pacific Conference Series; Astronomical Society of the Pacific: San Francisco, CA, USA, 2005, Volume 336, p. 25.

2. Savage, B.D.; Sembach, K.R. Interstellar Abundances from Absorption-Line Observations with the Hubble Space Telescope. Ann. Rev. Astron. Astrophys. 1996, 34, 279-330. [CrossRef]

3. Lodders, K. Solar System Abundances and Condensation Temperatures of the Elements. Astrophys. J. 2003, 591, 1220-1247. [CrossRef]

4. Kramida, A.; Ralchenko, Y.; Reader, J.; NIST ASD Team. NIST Atomic Spectra Database (Ver. 5.5.6); National Institute of Standards and Technology: Gaithersburg, MD, USA, 2018. Available: https:/ / physics.nist.gov/ asd (accessed on 30 May 2018).

5. Peck, E.R.; Reeder, K. Dispersion of Air. J. Opt. Soc. Am. 1972, 62, 958. [CrossRef]

6. Martin, W.C.; Kaufman, V.; Musgrove, A. A Compilation of Energy Levels and Wavelengths for the Spectrum of Singly-Ionized Oxygen (OII). J. Phys. Chem. Ref. Data 1993, 22, 1179-1212. [CrossRef]

7. Seaton, M.J.; Yan, Y.; Mihalas, D.; Pradhan, A.K. Opacities for Stellar Envelopes. Mon. Not. R. Astron. Soc. 1994, 266, 805. [CrossRef]

(c) 2018 by the authors. Licensee MDPI, Basel, Switzerland. This article is an open access article distributed under the terms and conditions of the Creative Commons Attribution (CC BY) license (http://creativecommons.org/licenses/by/4.0/). 